\title{
High-order extended finite element method for cracked domains
}

\author{
Patrick Laborde $^{1, \ddagger}$, Julien Pommier ${ }^{2, \S}$, Yves Renard ${ }^{2, *, \dagger}$ and Michel Salaün ${ }^{3, \mathbb{I}}$ \\ ${ }^{1}$ MIP, CNRS UMR 5640, UPS Toulouse 3, 118 route de Narbonne, 31062 Toulouse cedex 4, France \\ ${ }^{2}$ MIP, CNRS UMR 5640, INSAT, Complexe scientifique de Rangueil, 31077 Toulouse, France \\ ${ }^{3}$ ENSICA, 1 pl. Emile Blouin, 31056 Toulouse cedex 5, France
}

\begin{abstract}
SUMMARY
The aim of the paper is to study the capabilities of the extended finite element method (XFEM) to achieve accurate computations in non-smooth situations such as crack problems. Although the XFEM method ensures a weaker error than classical finite element methods, the rate of convergence is not improved when the mesh parameter $h$ is going to zero because of the presence of a singularity. The difficulty can be overcome by modifying the enrichment of the finite element basis with the asymptotic crack tip displacement solutions as well as with the Heaviside function. Numerical simulations show that the modified XFEM method achieves an optimal rate of convergence (i.e. like in a standard finite element method for a smooth problem). Copyright (C) 2005 John Wiley \& Sons, Ltd.
\end{abstract}

KEY WORDS: fracture; finite elements; extended finite element method; rate of convergence

\section{INTRODUCTION}

Computer simulation of fracture processes remains a challenge for many industrial modelling problems (cracking in concrete, delamination in composite materials, fatigue crack in adhesive bonded joints, etc.) [1-3]. In a classical finite element method, the non-smooth displacement near the crack tip is captured by refining the mesh locally. The number of degrees of freedom may drastically increase, especially in three-dimensional applications. Moreover, the incremental computation of a crack growth needs frequent remeshings. Reprojecting the solution on the updated mesh is not only a costly operation but also it may have a troublesome impact on the quality of results. Classical finite element methods have achieved their limit ability for solving fracture mechanics problems.

\footnotetext{
*Correspondence to: Yves Renard, MIP, CNRS UMR 5640, INSAT, Complexe scientifique de Rangueil, 31077 Toulouse, France.

†E-mail: renard@insa-toulouse.fr

*E-mail: laborde@mip.ups-tlse.fr

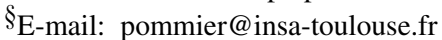

IE-mail: msalaun@ensica.fr
} 
To avoid these computational difficulties, a new approach to the problem consists in taking into account the a priori knowledge on the exact solution. Adding the asymptotic crack tip displacement solution to the finite element basis seems to be a somewhat early idea. Strang and Fix [4] applied this approach for the Laplace operator where the singular enrichments were truncated by a polynomial cut-off function. Babuška et al. [5-7] considered a partition of unity principle in conjunction with the finite element method to conciliate a local approximability property together with a global continuity condition. Mathematical results are presented to establish this new approach. The PUFEM method enables to use locally non-classical shape functions incorporated into the finite element basis remaining within the framework of conformal methods. Following these ideas, numerical works have been carried out [8-10]. A more general presentation can be found in Reference [11].

Moës et al. [12] introduced a numerical methodology which has been developed in the last few years by the name of XFEM-extended finite element method. This is an improvement of methods in Reference [13] where curved cracks were treated by mapping the straight crack enriched field. In Reference [12], not only finite elements are enriched with the asymptotic crack tip displacement solutions, but also with a step function which takes into account the jump of the displacement across the crack. Then, the finite element mesh can be defined independent of the crack geometry. The partition of unity chosen to localize the enrichment functions is linked to the mesh and is generally defined using linear shape functions. Several recent works have shown the efficiency of this method for two- or three-dimensional fracture problems [14-22].

An advantage of the XFEM method is to obtain more accurate numerical results than classical finite element one. However, the rate of convergence is not optimal with respect to the mesh parameter $h$. This rate is lower than it is expected with classical finite element method for a smooth problem [21]. In the present work, our aim is to propose some improvements of the XFEM method in order to obtain the optimal accuracy. An outline of this paper is as follows.

In Section 2, we define the model problem considered throughout the paper, which is relative to the equilibrium of a cracked body in plane linear elasticity.

Section 3 is devoted to the classical XFEM method. Its main features are briefly recalled (Section 3.1). A difficulty comes from the numerical integration of non-smooth functions. An 'almost polar integration' rule is introduced and its numerical efficiency is verified (Section 3.2). Then, we test the abilities of the XFEM method on the model problem, and the error curves are presented for a polynomial basis of degree 1,2 or 3 . It can be seen that the rate of convergence remains of order $\sqrt{h}$ only, in agreement with Stazi et al. [21] (Section 3.3). Some aspects of this unsatisfactory behaviour are analysed. First, the interpolation error in the XFEM method is not optimal (in comparison to the interpolation error given by the non-enriched finite element method for a smooth function). The reason is suggested with a simpler analogous onedimensional example. Second, adding the functions of enrichment to the finite element basis leads to a locally non-unisolvent method. Third, the discontinuity of the displacement field along the crack has to be represented in a sufficiently accurate way using the finite element basis as partition of unity (Section 3.4).

In Section 4, we introduce a first improvement motivated by the previous error analysis. In the classical XFEM method, only the nodes the nearest to the crack tip are enriched; consequently the support of the additional basis functions vanishes when $h$ goes to zero. An alternative strategy consists in enriching a whole fixed area around the crack tip independent of $h$. The expected optimal rate of convergence is nearly reached as it can be seen from the presented numerical tests. 
In Section 5, we improve the previous modification in order to decrease the number of unknowns and the condition number. A bonding condition is introduced on the enrichment area around the crack tip. Here, for each singular shape function, the equality of the corresponding degrees of freedom is prescribed (Section 5.1). Unfortunately, the numerical tests show a reduction of the rate of convergence, even though the condition number is significantly improved (Section 5.2). Analysing the difficulty from a mathematical point of view in a one-dimensional framework, we notice that the lack of accuracy comes from the elements in the transition layer, at the boundary of the enriched zone (Section 5.3). According to the original PUFEM method, let a partition of unity be defined independently of the mesh from an overlapping of the domain by two patches, the crack tip lying in the interior of one of them. Then we can prove that the error of convergence is optimal (Section 5.4). It may be seen also that the contribution to the error estimate of the intersection between the two patches does not have a significant influence. So it is quite natural to consider the limit case where the two patches constitute a partition of the domain. Then, the enrichment of the finite element basis leads to a non-conformal method, and one has to assign a matching condition at the interface between the two patches. Numerical tests show that a nodal matching condition supplies the optimal convergence rate. Let us notice that this improvement is achieved with a slight decrease of the size of the approximation problem compared to the classical XFEM method (Section 5.5). The more the approximation basis is fitted to the exact solution, the more an enriched finite element method is accurate.

In Section 6, we examine the performances of the previous modified XFEM method for solving a different problem. Here, the exact solution includes the second term in the asymptotic expansion of the solution to the previous model problem.

Finally, in Section 7, we focus on the computation of the stress intensity factors and we present the corresponding curves of convergence.

\section{MODEL PROBLEM}

A linear elasticity bidimensional problem is considered, with an isotropic homogeneous material, on a cracked domain $\Omega$ (see Figure 1 ). On the boundary of the non-cracked domain $\bar{\Omega}$, a Dirichlet condition is applied on $\Gamma_{\mathrm{D}}$ and a Neumann condition is prescribed on $\Gamma_{\mathrm{N}}$. The crack is denoted by $\Gamma_{\mathrm{C}}$ such that $\Gamma_{\mathrm{D}}, \Gamma_{\mathrm{N}}$ and $\Gamma_{\mathrm{C}}$ is a partition of $\partial \Omega$.

The space of admissible displacements is $\mathscr{V}=\left\{v \in H^{1}\left(\Omega ; \mathbb{R}^{2}\right) ; v=0\right.$ on $\left.\Gamma_{\mathrm{D}}\right\}$, and the equilibrium problem is written:

$$
\text { find } u \in \mathscr{V} \text { s.t. } a(u, v)=l(v) \quad \forall v \in \mathscr{V}
$$

where

$$
\begin{aligned}
a(u, v) & =\int_{\Omega} \sigma(u): \varepsilon(v) \mathrm{d} x \\
l(v) & =\int_{\Omega} f \cdot v \mathrm{~d} x+\int_{\Gamma_{\mathrm{N}}} g \cdot v \mathrm{~d} \Gamma \\
\sigma(u) & =\lambda \operatorname{tr} \varepsilon(u) I+2 \mu \varepsilon(u)
\end{aligned}
$$






Figure 1. A cracked domain.

with $\sigma(u)$ the stress tensor, $\varepsilon(u)$ the linearized strain tensor, $\lambda>0$ and $\mu>0$ the Lamé coefficients, $g$ and $f$ the given force densities on $\Omega$ and $\Gamma_{\mathrm{N}}$, respectively, and assuming a traction free condition on the crack. The source term $f$ is supposed to be sufficiently smooth, i.e. on a neighbourhood $\omega_{\mathrm{C}}$ of the crack tip, we assume that $f \in H^{m}\left(\omega_{\mathrm{C}} ; \mathbb{R}^{2}\right)$ for some $m \geqslant 0$. Hence the solution $u$ can be written as a sum of a singular part and a regular part such that:

$$
u-\sum_{n=0}^{m} r^{1 / 2+n}\left(c_{n}^{1} w_{n}^{1}+c_{n}^{2} w_{n}^{2}\right) \in H^{m+2}\left(\omega_{\mathrm{C}} ; \mathbb{R}^{2}\right)
$$

(See Reference [23]), where $r$ denotes the distance from the crack tip. The normal (resp. tangential) component of the functions $w_{n}^{1}$ (resp. $w_{n}^{2}$ ) is discontinuous along the crack. They both correspond to the well known I and II opening modes for a bidimensional crack.

The asymptotic displacement at the crack tip for these two modes is given in polar coordinates relatively to the crack tip by (see Figure 2)

$$
\begin{aligned}
& u_{\mathrm{I}}=\frac{K_{\mathrm{I}}}{E} \sqrt{\frac{r}{2 \pi}}\left(\begin{array}{c}
\cos \frac{\theta}{2} \\
\sin \frac{\theta}{2}
\end{array}\right)(a+b \cos \theta) \\
& u_{\mathrm{II}}=\frac{K_{\mathrm{II}}}{E} \sqrt{\frac{r}{2 \pi}}(1+v)\left(\begin{array}{c}
\sin \frac{\theta}{2}(c+2+\cos \theta) \\
\cos \frac{\theta}{2}(2-c-\cos \theta)
\end{array}\right)
\end{aligned}
$$

where $K_{\mathrm{I}}$ and $K_{\mathrm{II}}$ are the stress intensity factors (see for instance Reference [24]), and

$$
a=2+\frac{2 \mu}{\lambda+2 \mu}, \quad b=-2 \frac{\lambda+\mu}{\lambda+2 \mu}, \quad c=\frac{\lambda+3 \mu}{\lambda+\mu}
$$

The functions $u_{\mathrm{I}}$ and $u_{\mathrm{II}}$ belong to $H^{3 / 2-\varepsilon}\left(\Omega ; \mathbb{R}^{2}\right)$ for any $\varepsilon>0$ (see Reference [23], Chapter 4). 




Figure 2. Polar co-ordinates relatively to the crack tip.

\section{THE CLASSICAL XFEM}

\subsection{Description}

The basic idea of XFEM is to enrich a classical finite element space with some additional functions. These functions are built as the product of global enrichment functions with some finite element functions.

One considers a Lagrange finite element method of order $k$ defined on a regular triangulation of the uncracked domain $\bar{\Omega}$. The $P_{k}$ (vector valued) basis functions are denoted $\varphi_{1} \ldots \varphi_{N}$, where $P_{k}$ stands for the set of order $k$ polynomials.

The XFEM enriched space is then

$$
\mathscr{V}^{h}=\left\{v^{h}=\sum_{i=1}^{N} a_{i} \varphi_{i}+\sum_{i \in I_{H}} b_{i} H \psi_{i}+\sum_{i \in I_{F}} \sum_{j=1}^{4} c_{i j} F_{j} \psi_{i}: a_{i} \in \mathbb{R}, \quad b_{i} \in \mathbb{R}, \quad c_{i j} \in \mathbb{R}\right\}
$$

where $\psi_{i}$ are the basis functions of the vector $P_{1}$ finite element method (used for the partition of unity). Here, $I_{H}$ is the set of the degrees of freedom indices enriched with the global discontinuous step function $H(x)=\operatorname{sign}\left(d_{\Gamma_{\mathrm{C}}}(x)\right)$, denoting $d_{\Gamma_{\mathrm{C}}}$ a signed distance from the crack. Similarly, $I_{F}$ is the set of the degrees of freedom indices enriched with the singular functions $F_{j}(x)$ defined as follows (in polar co-ordinates relatively to the crack tip):

$$
\begin{aligned}
& F_{1}(r, \theta)=\sqrt{r} \sin \frac{\theta}{2} \\
& F_{2}(r, \theta)=\sqrt{r} \cos \frac{\theta}{2} \\
& F_{3}(r, \theta)=\sqrt{r} \sin \frac{\theta}{2} \cos \theta \\
& F_{4}(r, \theta)=\sqrt{r} \cos \frac{\theta}{2} \cos \theta
\end{aligned}
$$




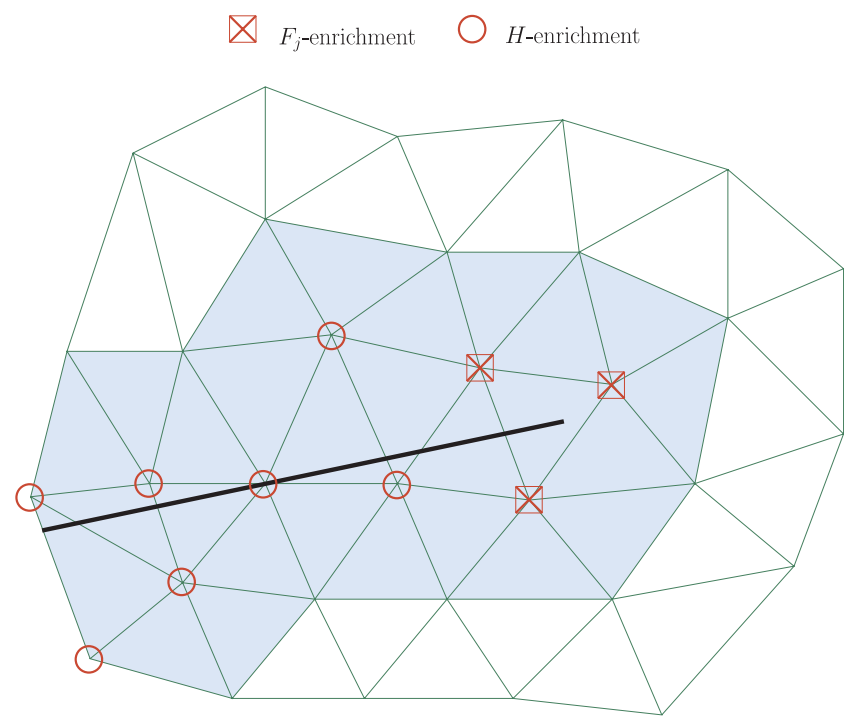

Figure 3. Selection of the enriched nodes.

The determination of $I_{H}$ and $I_{F}$ is done according to the following rules (see Figure 3 and Reference [12]):

- $I_{H}$ is the set of nodes whose basis functions support is entirely splitted by the crack,

- $I_{F}$ is the set of nodes which contain the crack tip in the (interior of the) support of their basis function.

\subsection{Numerical integration}

There are two difficulties for the integration of XFEM functions: the discontinuity along the crack, and the singularity at the crack tip. If the discontinuity is not taken into account when the numerical integration is performed, this may lead to bad numerical results, and even to a non-invertible set of equations if the integration points 'miss' the discontinuity.

The usual rule is to perform a simple splitting of the triangles crossed by the crack such that the resulting set of triangles does not cross the crack [25] (see Figure 4). Note that this does not require a general mesh refinement procedure: flat triangles are not a problem.

For the elements that contain the crack tip, a special care has to be taken. The discontinuity is still present, but there is also a singularity. So, simply splitting the triangles may lead to poor numerical results if the integration method is not precise enough. A naive solution is to refine locally each splitted triangle, until a good estimate of the integrals is achieved. Unfortunately, the computational cost increases a lot, since a very large number of integration points (ten thousands or more) may be required.

However, if one looks at the expressions of the elementary integrals,

$$
\int_{T} \nabla\left(F_{i} \psi_{j}\right) \cdot \nabla\left(F_{k} \psi_{l}\right) \mathrm{d} x
$$




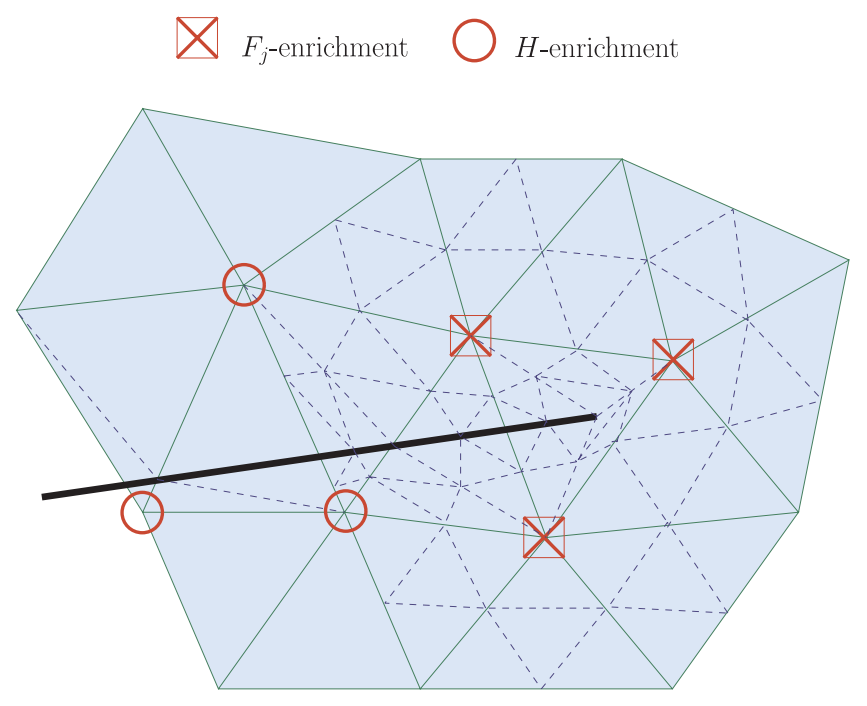

Figure 4. Local refinement for quadrature rules.

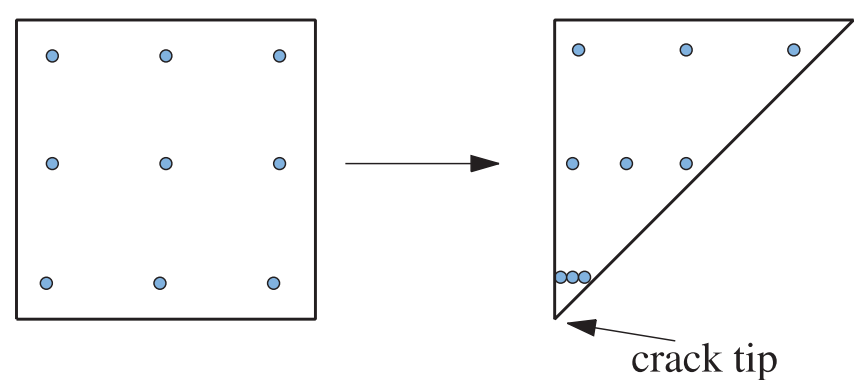

Figure 5. Transformation of an integration method on a square into an integration method on a triangle for crack tip functions.

one notices that expressing this integral in polar co-ordinates will cancel the $r^{-1 / 2}$ singularity of $\nabla F_{i}(x)$. Hence, the following integration method gives excellent results with a very low number of integration points when it is used on the sub-triangles having the crack tip as a vertex (keeping a classical Gauss quadrature on the other sub-triangles).

The geometric transformation

$$
\tau:\left(\begin{array}{l}
x_{1} \\
x_{2}
\end{array}\right) \rightarrow\left(\begin{array}{c}
x_{1} x_{2} \\
x_{2}
\end{array}\right)
$$

maps the unit square onto a triangle (Figure 5). Using this transformation, it is possible to build a quadrature rule on the triangle from a quadrature rule on the unit square. The new 


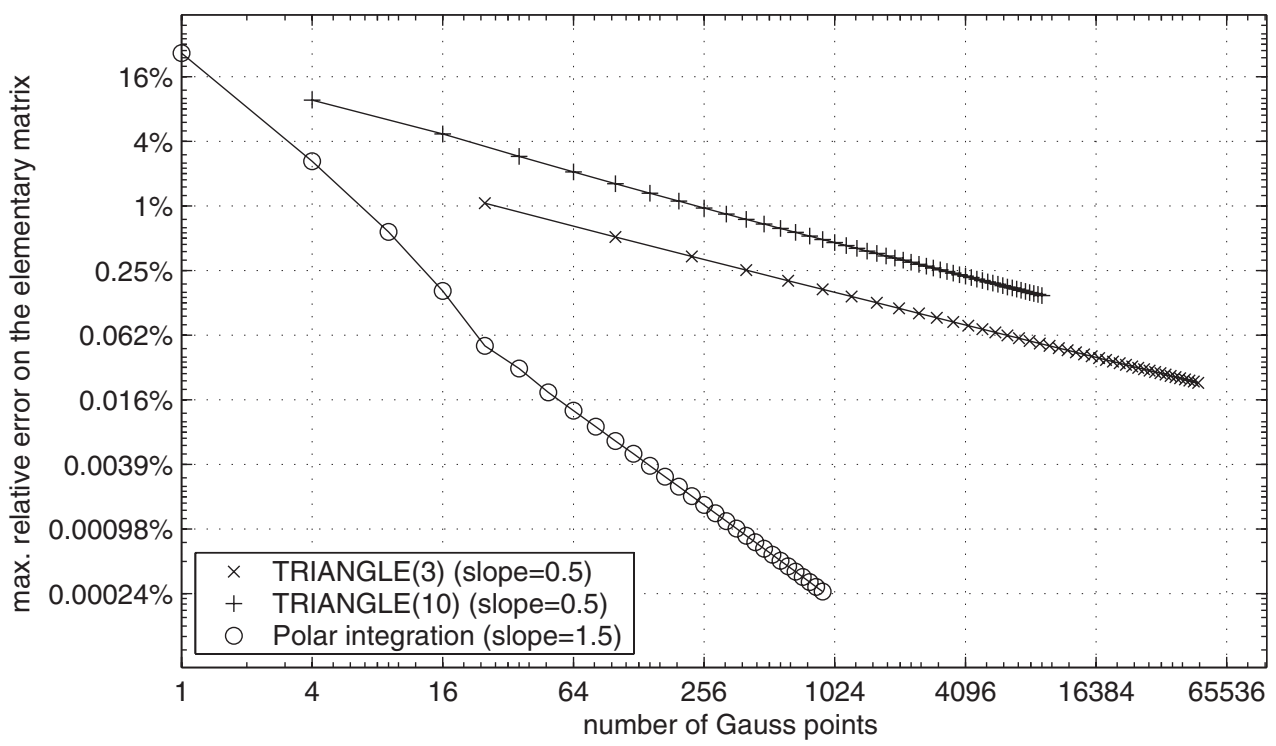

Figure 6. Comparison of integration methods (uniform refinement with order 3 Gauss method and order 10 Gauss method, and almost polar integration).

integration points $\bar{\xi}$ and their weights $\bar{\eta}$ are obtained from those of the original quadrature rule with

$$
\begin{aligned}
& \bar{\xi}=\tau(\xi) \\
& \bar{\eta}=\eta \operatorname{det}(\nabla \tau)
\end{aligned}
$$

This quadrature will be called in the following the 'almost polar integration'. Independently, in Reference [26], an integration method which also uses the non-singular characteristic of the polar form of the integrals has been developed.

The performances of the classical refined integration and the almost polar integration are compared by computing a XFEM elementary matrix.

The reference elementary matrix is computed on a very refined subdivision near the singularity point. Figure 6 presents the relative error between this reference elementary matrix and a computation of the elementary matrix with the following different strategies:

- using a regular refinement of the triangle, and a fixed integration rule on each refined triangle (of order 3 and 10);

- using the almost polar integration method, without any additional refinement, but for Gauss quadratures of increasing order.

This shows that the almost polar integration approach offers an important gain. In practice, 25 Gauss points were enough for the most accurate convergence test we have done. 

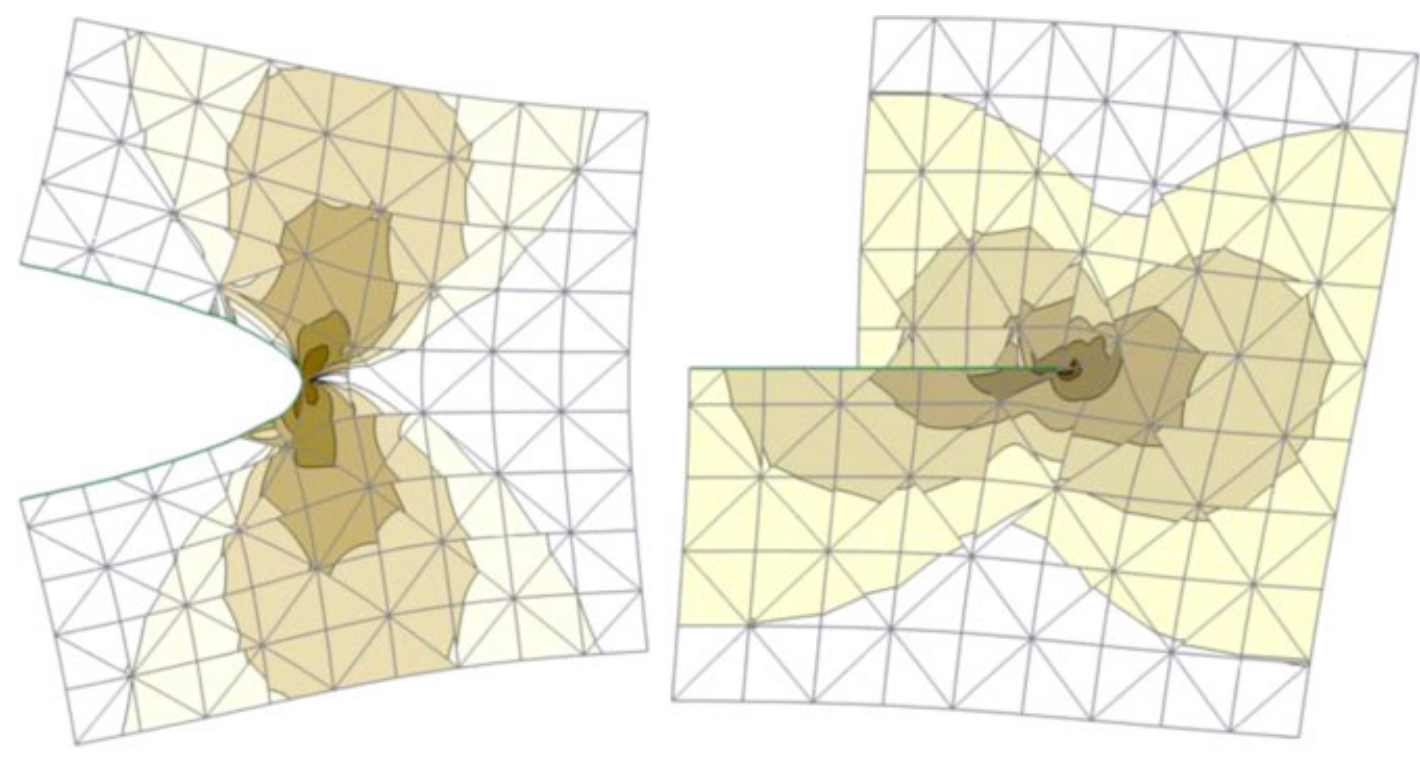

Figure 7. $P_{2}$ XFEM solutions for mode I and mode II problems (with contour levels of Von Mises stress).

\subsection{Convergence tests}

The two test cases presented (see Figures 7 and 8) are chosen such that mode I (resp. mode II) crack displacement is the exact solution. This is ensured via adequate (non-homogeneous) Dirichlet conditions on the domain boundary. The domain is $\bar{\Omega}=[0,5] \times[-2.5,2.5]$ and the considered crack is the line segment $\Gamma_{C}=[0,2.5] \times\{0\}$. The domain is discretized with a regular mesh.

As suggested in Reference [21], the $\psi_{i}$ functions of Equation (4) are taken as the basis functions of a $P_{1}$ finite element method, even for $P_{2}$ XFEM and $P_{3}$ XFEM.

The convergence curves, presented in Figure 9, are very similar to the ones obtained by Stazi et al. [21]. One can note that, although the energy error is lower with XFEM than without enrichment, XFEM does not improve the convergence rate which is $O(\sqrt{h})$.

\subsection{Interpretation of the previous results}

It may seem surprising that XFEM does not improve the convergence rate of the finite element method. Since the singularity is added into the finite element space, and the non-singular part is sufficiently smooth, one would expect to get the error rate of the classical finite elements, i.e. $\left\|u-u_{h}\right\|_{H^{1}}=O\left(h^{k}\right)$ for $P_{k}$ elements.

3.4.1. One-dimensional analysis. In order to analyse this relatively poor convergence rate, let us consider a one-dimensional case. Even though the problem is different, it seems to us that the conclusions are sufficiently clear to explain what happens in the two-dimensional case. Let us assume that one wants to approximate on the domain $[0,1]$ the following 

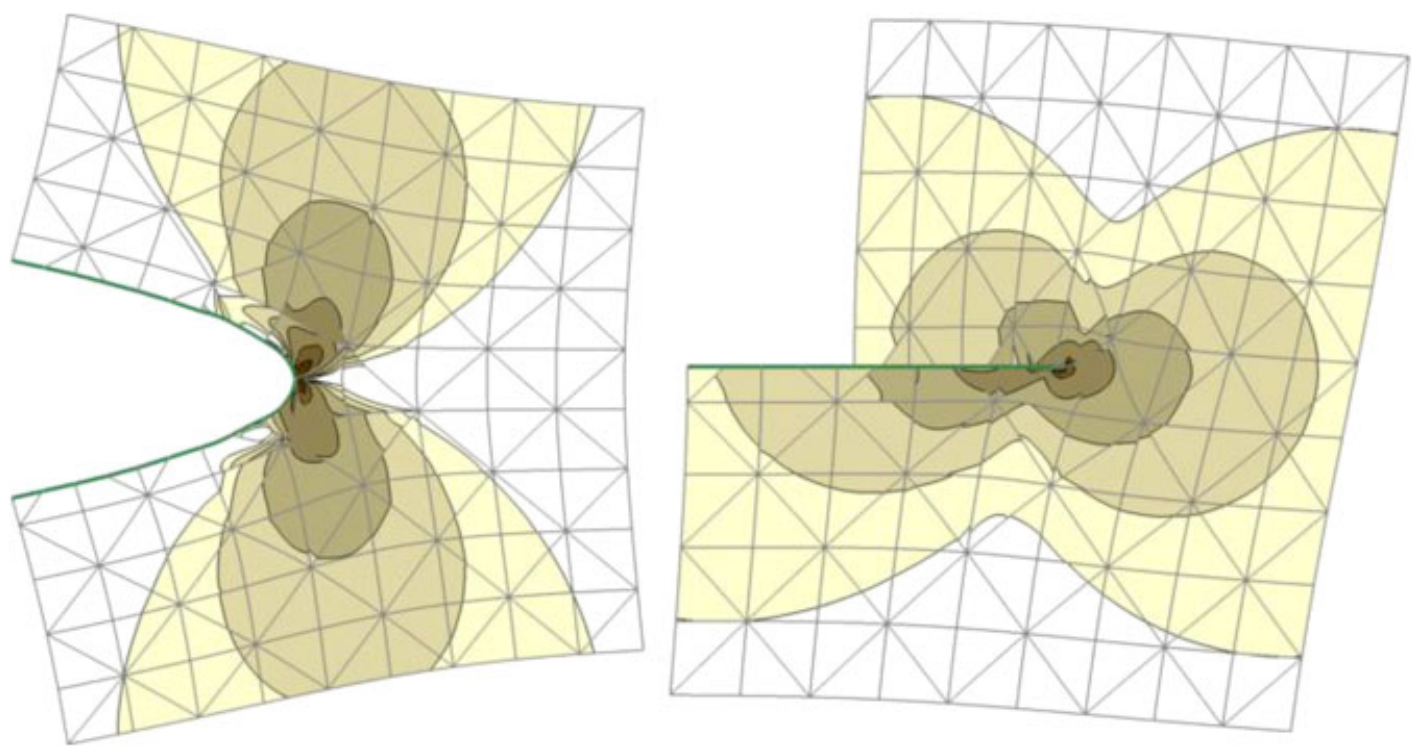

Figure 8. $P_{3}$ XFEM solutions for mode I and mode II problems (with contour levels of Von Mises stress).

function:

$$
G(x)=x(\ln (x)-1)
$$

which is in the Sobolev space $H^{3 / 2-\varepsilon}(0,1)$ for any $\varepsilon>0$ (the function $\sqrt{x}$ is not in $H^{1}(0,1)$, so it is not relevant for our purpose).

If one considers the classical Lagrange $P_{1}$ approximation on the domain $[0,1]$ divided in $N$ intervals of length $h=1 / N$, then the square of the $H^{1}(0,1)$ semi-norm of the interpolation error on an interval is

$$
\begin{aligned}
e_{i}^{2} & =\int_{i h}^{(i+1) h}\left(\frac{\mathrm{d}}{\mathrm{d} x}\left(G(x)-G((i+1) h)\left(\frac{x}{h}-i\right)-G(i h)\left(i+1-\frac{x}{h}\right)\right)\right)^{2} \mathrm{~d} x \\
& =h\left(1-(i+1) i \ln ^{2}\left(1+\frac{1}{i}\right)\right)
\end{aligned}
$$

Hence, denoting $G_{h}$ the interpolate of $G$, one has

$$
\left\|G-G_{h}\right\|_{H^{1}(0,1)}^{2}=\sum_{i=1}^{N} e_{i}^{2}=C^{2} h
$$

with $C^{2}=\sum_{i=0}^{+\infty}\left(1-(i+1) i \ln ^{2}(1+(1 / i))\right)$. It is easy to verify that this series converges, and $C^{2} \approx 2.70$. One can conclude that the $H^{1}(0,1)$ semi-norm of the interpolation error is close to $C \sqrt{h}$. This is exactly what is expected for a $P_{1}$ finite element method with a solution which has this kind of regularity. 

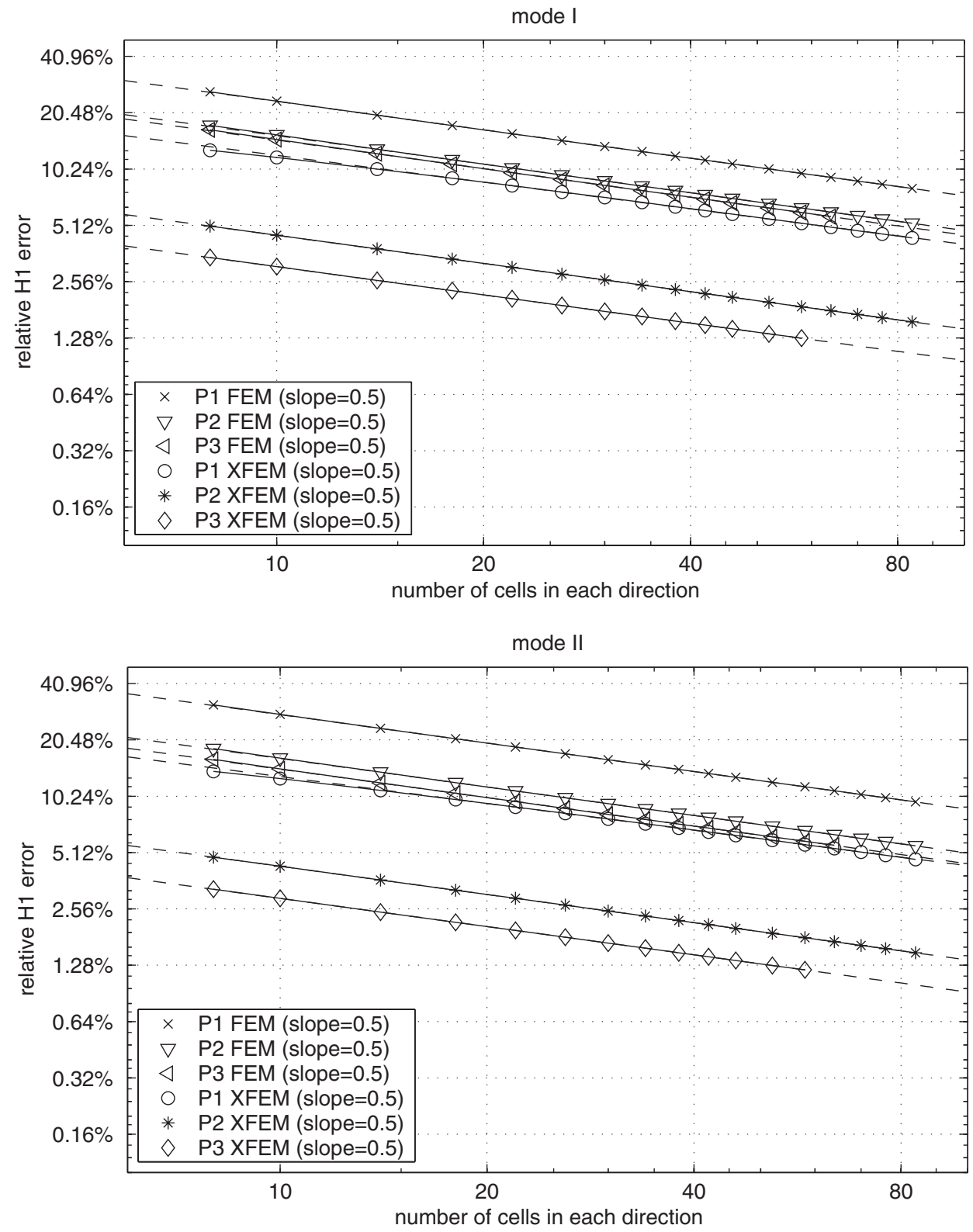

Figure 9. Convergence comparison of classical finite elements and XFEM for mode I and mode II problems.

Let us see now the behaviour of the classical XFEM based on the $P_{1}$ finite element method enriched with the singular function $G(x)$, but only on the first node of the first interval $[0, h]$. At best, the interpolation error will vanish on the first interval and will be unchanged on 
the others. Thus, the $H^{1}(0,1)$ semi-norm of the interpolation error is close to $\bar{C} \sqrt{h}$, with $\bar{C}^{2} \geqslant C^{2}-1$. The error is smaller, but the order of convergence is unchanged. Of course, this reasoning works independent of the degree of the finite element method, thus the classical XFEM will be convergent with an order $O(\sqrt{h})$ independently of this degree.

A solution to increase the convergence rate is to consider a constant area of enrichment (i.e. independent of $h$ ). This is one of the improvements we will propose.

3.4.2. XFEM is locally non-unisolvent. Implementing the classical XFEM, one may remark that the elementary stiffness matrices have seven vanishing eigenvalues, while classical elementary stiffness matrices have normally three, corresponding to two free translations and one free rotation. This is due to the fact that XFEM is non-unisolvent in the sense that one has

$$
\begin{aligned}
& \phi_{2}\left(F_{1}-F_{4}\right)+\phi_{1} F_{3}=0 \\
& \phi_{2}\left(F_{3}-F_{2}\right)+\phi_{1} F_{4}=0
\end{aligned}
$$

with $\phi_{i}$ is a $P_{1}$ basis,

$$
\phi_{1}(x, y)=x, \quad \phi_{2}(x, y)=y \quad \text { and } \quad \phi_{3}(x, y)=1-x-y
$$

Since the XFEM basis is a linear combination of $\phi_{i} F_{j}$ for each component $u^{1}, u^{2}$, this leads to four linear relations between the XFEM shape functions and thus to four vanishing eigenvalues in the elementary stiffness matrix. Moreover, this remark holds for a $P_{1}$ finite element method used as partition of unity. If a $P_{2}$ finite element method is used, there are six relations of this kind, so 12 additional vanishing eigenvalues. This is one of the reasons we only use the $P_{1}$ partition of unity for the singular functions. Let us remark that the same choice is also made by Stazi et al. [21].

3.4.3. Low degree for the step function. In the definition of the XFEM discrete space (4), the step function $H$ is multiplied by the shape functions of a $P_{1}$ finite element method $\psi_{i}$. Thus, the displacement jump will be represented as a piecewise linear function along the crack. This is of course incompatible with an higher order representation of the displacement. In order to have an optimal convergence rate, the partition of unity used for the function $H$ has to be of the same degree as the finite element method used to approximate the displacement. So the term $\sum_{i \in I_{H}} b_{i} H \psi_{i}$ in (4) has to be replaced by $\sum_{i \in I_{H}^{\prime}} b_{i} H \varphi_{i}$, where $I_{H}^{\prime}$ is the appropriate set of degrees of freedom. This will be done in the following (still denoting $I_{H}$ instead of $I_{H}^{\prime}$ ). This idea was also mentioned in Reference [12], although not fully tested.

\section{XFEM WITH A FIXED ENRICHMENT AREA (XFEM-f.a.)}

\subsection{Principle}

As it was previously observed on the convergence curves (see Figure 9), the classical XFEM method does not improve the order of convergence of a usual finite element method. The convergence rate remains to $O(\sqrt{h})$, which is the expected order, because of the nature of the crack tip singularity. Naturally, the level of error, in energy norm, is better for XFEM but 


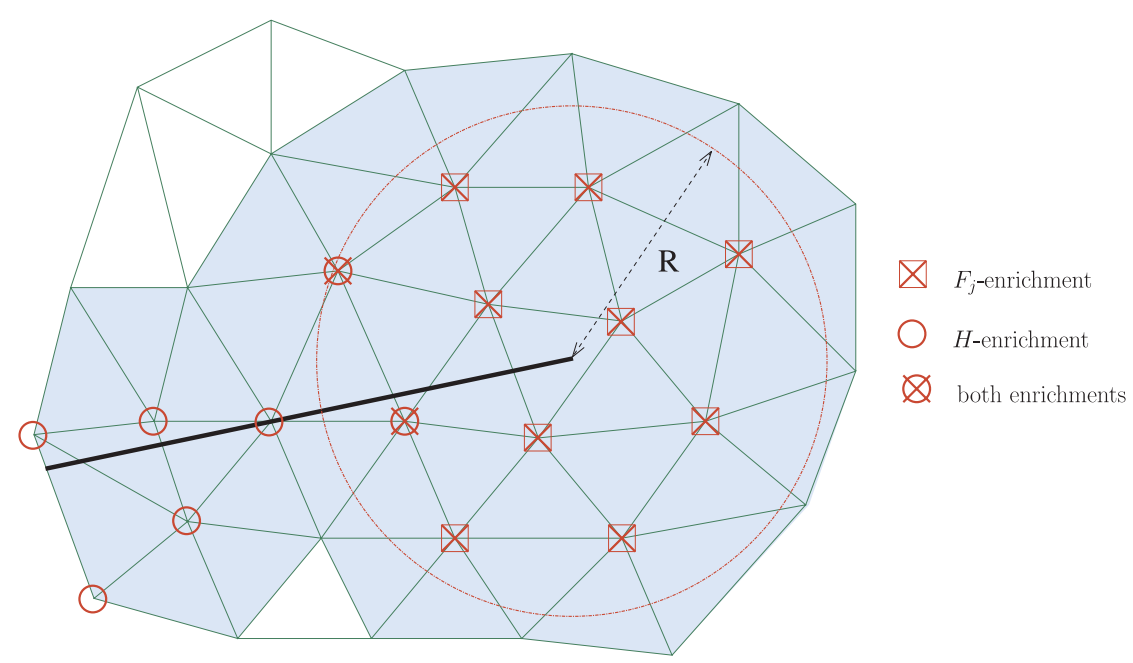

Figure 10. Enrichment with a fixed area for singular functions.

one could expect a better behaviour. Our simplified analysis (see Section 3.4.1) shows that a necessary condition for optimality is to have an enrichment area with a size independent of the mesh parameter $h$. This is the idea we develop in this section.

Let us introduce $B\left(x_{0}, R\right)$ the disk whose centre is the crack tip $x_{0}$. Its radius $R$ is a strictly positive number, independent of $h$, chosen small enough such that $B\left(x_{0}, R\right)$ is contained in the non-cracked domain $\bar{\Omega}$. For the further convergence tests, this radius $R$ will be fixed to $1 / 10$ th of the domain dimension. Then, we introduce the set $I(R)$ of the degrees of freedom which belong to triangles contained in $B\left(x_{0}, R\right)$ (see Figure 10). We can now define the new finite element space $\mathscr{V}_{R}^{h}$ as

$$
\mathscr{V}_{R}^{h}=\left\{v^{h}=\sum_{i=1}^{N} a_{i} \varphi_{i}+\sum_{i \in I_{H}} b_{i} H \varphi_{i}+\sum_{i \in I(R)} \sum_{j=1}^{4} c_{i j} F_{j} \psi_{i}: a_{i} \in \mathbb{R}, \quad b_{i} \in \mathbb{R}, \quad c_{i j} \in \mathbb{R}\right\}
$$

where we recall that the functions $\varphi_{i}$ (resp. $\psi_{i}$ ) are the shape functions of the $P_{k}$ finite element method (resp. $\left.P_{1}\right)$.

This improvement of the XFEM method has been developed independently in Reference [26].

\subsection{Convergence curves}

The convergence curves for mode I test problem are given in Figure 11. It shows that, for the different choices of the polynomials degrees, the convergence rates are close to the expected optimal ones, especially for sufficiently refined meshes (at least 16 cells in each direction). For mode II, we do not present the results because they are very similar to mode I. 


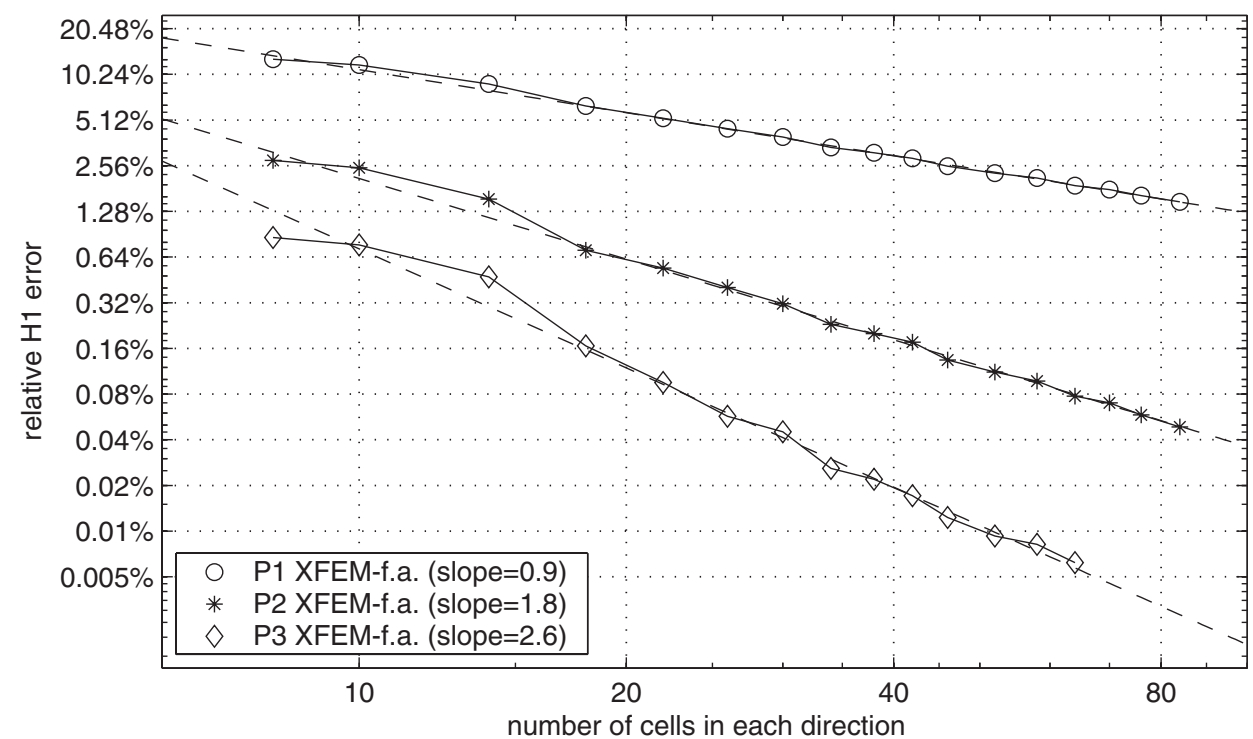

Figure 11. Convergence for $P_{k}$ XFEM with a fixed enrichment area (XFEM-f.a.): mode I problem.

Table I. Total number of degrees of freedom ( $N$ is the number of cells in each direction).

\begin{tabular}{|c|c|c|c|c|c|c|}
\hline$N$ & $P_{1}$ FEM & $P_{1}$ XFEM-f.a. & $P_{2}$ FEM & $P_{2}$ XFEM-f.a. & $P_{3}$ FEM & $P_{3}$ XFEM-f.a. \\
\hline 8 & 104 & 112 & 456 & 472 & 1064 & 1088 \\
\hline 30 & 1710 & 1926 & 6990 & 7236 & 15870 & 16146 \\
\hline 64 & 8000 & 9032 & 32320 & 33416 & 73024 & 74184 \\
\hline
\end{tabular}

\subsection{Remarks}

Even though the numerical results are very much improved, compared to the classical XFEM, this new approach introduces two drawbacks.

- Choosing a fixed enrichment area around the crack tip increases the total number of degrees of freedom. It is illustrated in Table I, where $N$ stands for the number of cells in each direction. Nevertheless, as the enrichment due to crack tip singularity uses first degree polynomial functions (see (7)) in all cases, the number of additional degrees of freedom does not depend too much on the degree of the polynomials, which are used for the approximation of the regular part of the solution. In particular, when we choose third order polynomials, the additional cost becomes rather negligible.

- The linear systems, associated to the finite element approximation, become more and more ill-conditioned (see Figure 12). One of the reasons may be the non-unisolvence of XFEM (see Section 3.4.2). This bad conditioning should explain the lack of optimality in the convergence rates (from 1.8 instead of 2 for $P_{2}$ to 2.6 instead of 3 for $P_{3}$, see Figure 11). Another explanation of this result may also be a problem in the transition layer (see Section 5.3). 


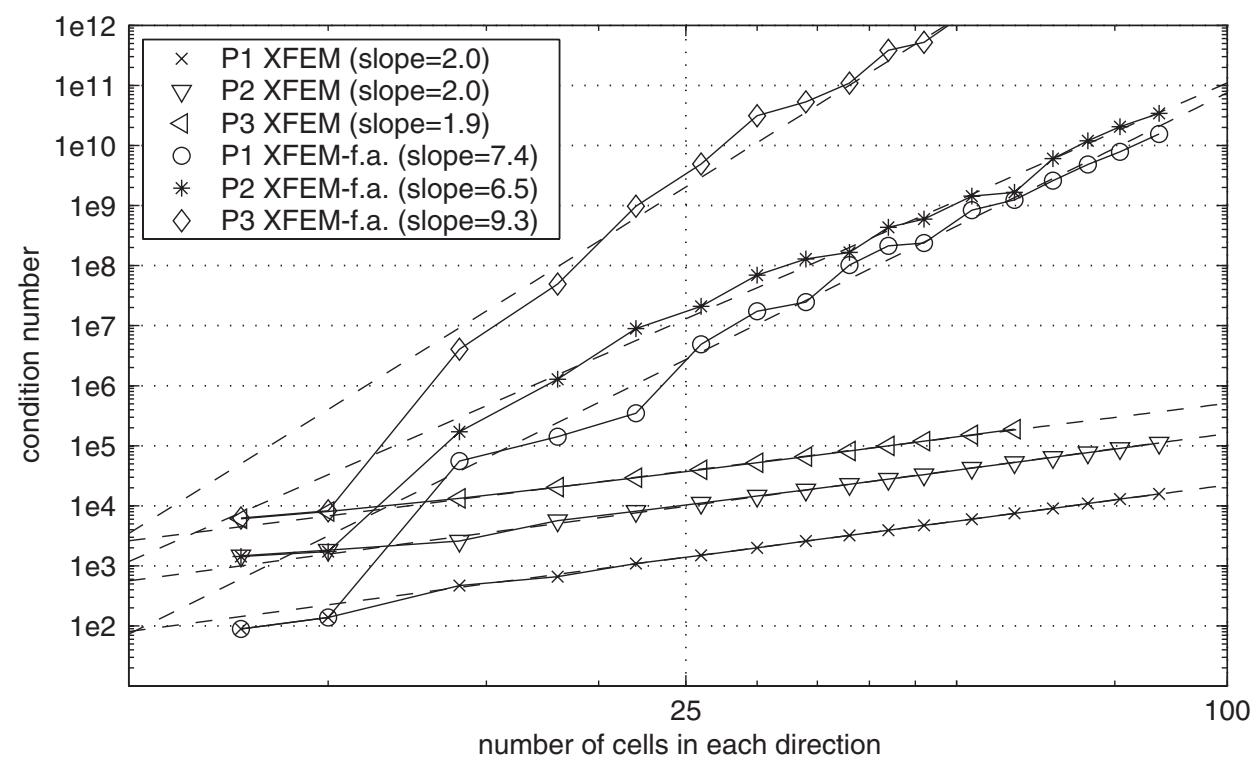

Figure 12. Condition numbers for mode I problem: comparison between XFEM with a fixed enrichment area (XFEM-f.a.) and the classical XFEM.

\section{DEGREES OF FREEDOM GATHERING (XFEM-d.g.) FOR THE SINGULAR FUNCTIONS}

\subsection{Description}

The basic idea of XFEM is to add the singular part of the exact solution in the finite element space. But, the singular functions are multiplied by finite element polynomials on a small family of nodes around the crack tip. Then, the singular functions live on a compact support vanishing as $h$ goes to zero. Unfortunately, the numerical experiments of the previous sections show that the enrichment area should have a size independent of the mesh parameter to expect optimal convergence results. Nevertheless, adding singular functions on all the nodes of a fixed disk around the crack tip results in a relative increase of the number of degrees of freedom and a huge increase of the condition number of the resulting linear system.

So, mixing the original ideas of XFEM (adding the singularities around the crack tip) and our numerical observations (enrichment area independent of the mesh parameter) leads us to the following strategy. We add the global functions $F_{j}(x) T_{R}(x)$, where $T_{R}(x)$ is the truncation function, given by

$$
T_{R}(x)=\sum_{i \in I(R)} \hat{\psi}_{i}(x)
$$

with $\hat{\psi}_{i}(x)$ the classical first degree scalar shape functions of the finite element method. Let us recall that, when we consider all the shape functions on an element, their sum is identically equal to one. So the function $T_{R}(x)$ is identically equal to 1 on all the triangles contained in the disk $B\left(x_{0}, R\right)$, and linearly decreases to 0 on the transition layer (see Figure 13). Let us remark that while the classical XFEM adds eight degrees of freedom for each enriched 


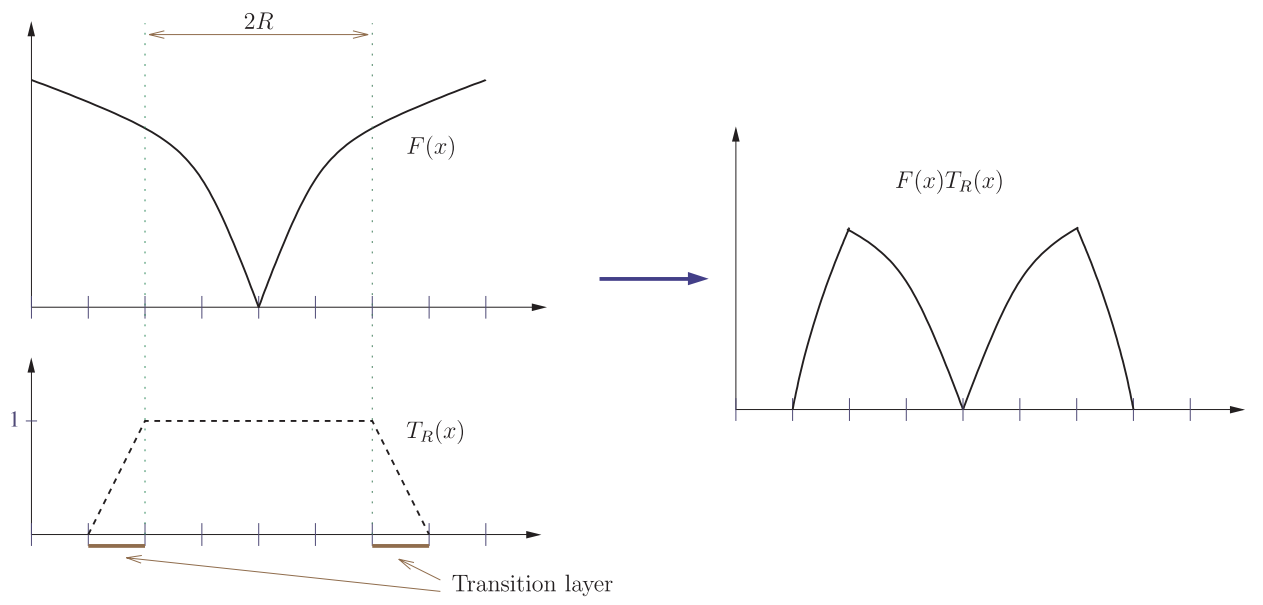

Figure 13. Truncation of an enrichment singular function $F$ (one-dimensional version).

node, the present method only adds eight degrees of freedom (four for each component of $u$ ), independent of the size of the enriched area. This additional cost is really negligible and makes conceivable the enrichment with more than four singular functions. Of course, the corresponding entries of the stiffness matrix are linked to each degree of freedom of the enriched area. But, since only eight lines/columns are filled, the impact on the performance of linear systems solvers is low.

The corresponding discrete space is given by

$$
\mathscr{V}_{G}^{h}=\left\{v^{h}=\sum_{i=1}^{N} a_{i} \varphi_{i}+\sum_{i \in I_{H}} b_{i} H \varphi_{i}+\sum_{j=1}^{4} c_{j}^{1} F_{j} T_{R} e_{1}+\sum_{j=1}^{4} c_{j}^{2} F_{j} T_{R} e_{2}: a_{i} \in \mathbb{R}, b_{i} \in \mathbb{R}, c_{j}^{k} \in \mathbb{R}\right\}
$$

where $e_{1}$ and $e_{2}$ is the canonical basis of $\mathbb{R}^{2}$.

\subsection{Numerical tests}

The convergence curves for mode I test problem are given in Figure 14. Rather surprisingly, these numerical tests exhibit an important lack of optimality on the convergence rates. This point is especially important for $P_{1}$ and $P_{2}$ polynomials. More precisely, we lose exactly half an order compared to optimality, which means $\sqrt{h}$, on all the curves, independently of the degree of the polynomials. The next section deals with an explanation of this phenomenon. To conclude this part, let us just observe that, despite the previous problem, the conditioning of the numerical problem is drastically improved by this technique (compare Figure 15 with Figure 12). 




Figure 14. Convergence of XFEM with d.o.f. gathering for mode I problem.

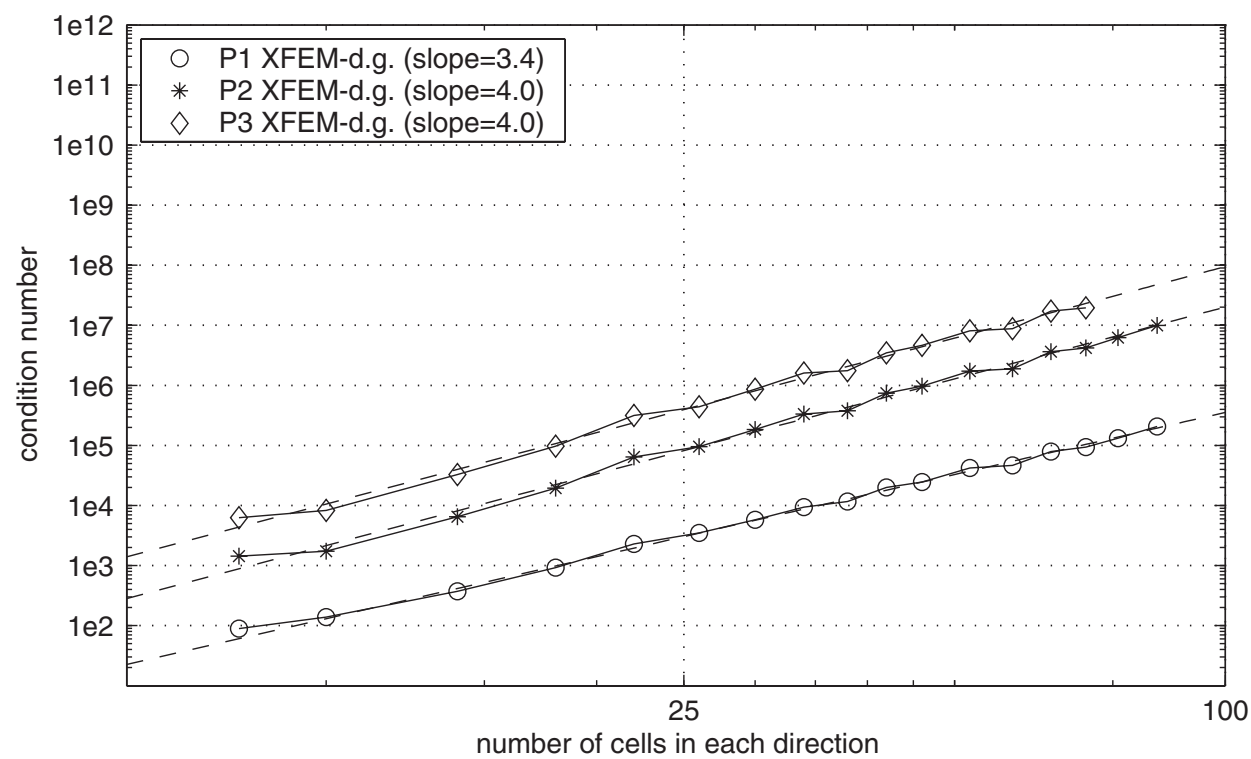

Figure 15. Condition numbers of XFEM with d.o.f. gathering for mode I problem. 


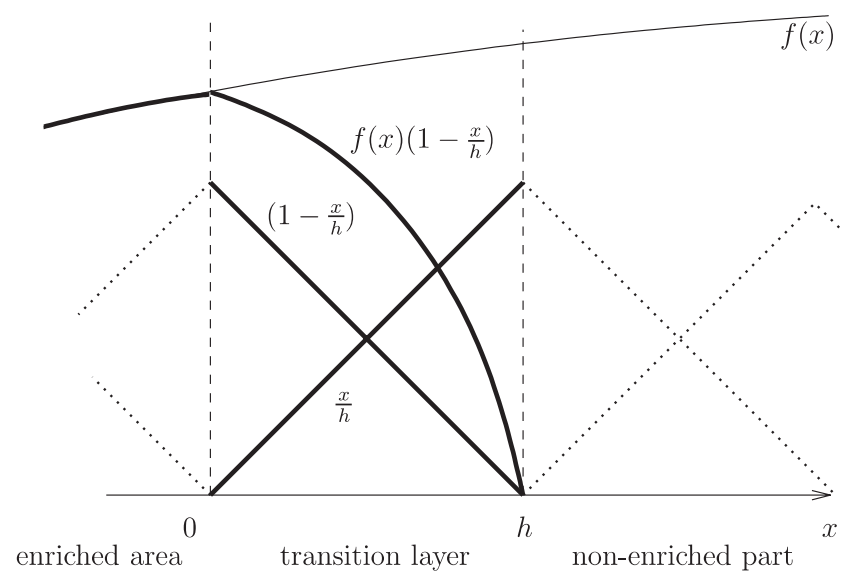

Figure 16. The transition layer for a one-dimensional XFEM-d.g.

\subsection{Analysis of the lack of optimal convergence}

For the sake of simplicity, we present a one-dimensional analysis of the interpolation error. The singular part of the solution is fully taken into account inside the enrichment area and, outside this area, the solution is regular and well approximated by the standard $P_{k}$ finite element method. Thus, the only potential problem comes from the transition layer between the enrichment area and the rest of the domain, i.e. the triangles partially enriched. We present the analysis for a $P_{1}$ and a $P_{2}$ XFEM. Let us remark that a different analysis of this kind of problem is done in Reference [22] where a specific reproducing condition is introduced. But this analysis cannot be straightforwardly applied to the present problem due to the non-polynomial singular functions.

5.3.1. $P_{1}$ analysis. We will analyse what happens in the transition layer in a one-dimensional case with an XFEM based on a piecewise linear finite element method. Assuming that the considered domain is $[-1 / 2,1 / 2]$, that the transition layer is represented by the interval $[0, h]$ and that only node 0 is enriched with $f(x)$ a singular function (The singularity of $f$ is supposed to be located in a point $x<0$ ). The shape functions on this (semi-enriched) interval are (see Figure 16):

$$
\left(1-\frac{x}{h}\right), \quad \frac{x}{h} \quad \text { and } \quad f(x)\left(1-\frac{x}{h}\right)
$$

Hence, on $[0, h]$, an interpolation will be written $f^{h}(x)=f(h)(x / h)+b(1-(x / h))+c f(x)$ $(1-(x / h))$ with $b+c f(0)=f(0)$. However, on $[-1 / 2,0]$, the interpolation space is $\mathscr{V}^{h}=$ $P_{1} \oplus\{\alpha f(x), \alpha \in \mathbb{R}\}$. As $f$ is singular, the only way to have an optimal convergence rate is to interpolate $f$ by itself. Hence at $x=0$, this implies that $c=1$ and then $b=0$. Finally, on $[0, h]$

$$
f^{h}(x)=\left(1-\frac{x}{h}\right) f(x)+\frac{x}{h} f(h)
$$


The interpolation error on this interval is given by

$$
f^{h}(x)-f(x)=\frac{x}{h}(f(h)-f(x))=\frac{x}{h}\left(f^{\prime}(x)(h-x)+f^{\prime \prime}(x) \frac{(h-x)^{2}}{2}\right)+O\left(h^{3}\right)
$$

so that on this transition layer one has

$$
\int_{0}^{h}\left|f^{h}(x)-f(x)\right|^{2} \mathrm{~d} x \leqslant C h^{3}
$$

where $C$ is a generic constant independent of $h$ (it depends of sup $\left|f^{\prime}\right|$ for this particular estimate). On other intervals $[n h,(n+1) h]$, not included in the enrichment area, the standard $P_{1}$ interpolation leads to

$$
\begin{aligned}
f^{h}(x)-f(x) & =\left(1-\frac{x-n h}{h}\right) f(n h)+\frac{x-n h}{h} f((n+1) h)-f(x) \\
& =\left(1-\frac{x-n h}{h}\right)(f(n h)-f(x))+\frac{x-n h}{h}(f((n+1) h)-f(x))
\end{aligned}
$$

thus

$$
\int_{n h}^{(n+1) h}\left|f^{h}(x)-f(x)\right|^{2} \mathrm{~d} x \leqslant C h^{5}
$$

(see more details in Reference [4]).

The $L^{2}$ norm on the global domain including the transition layer will be bounded by

$$
\left(\int_{-1 / 2}^{1 / 2}\left|f^{h}(x)-f(x)\right|^{2} \mathrm{~d} x\right)^{1 / 2} \leqslant C \sqrt{\left(\frac{N}{2}-1\right) h^{5}+h^{3}} \leqslant C h^{3 / 2}
$$

where $h=1 / N$. This is of course not optimal, since the $L^{2}$ interpolation error with a standard $P_{1}$ method is of order $h^{2}$ on a smooth function. What is lost seems to be the partition of unity effect of the standard $P_{1}$ method expressed by (9) which cannot be used on the transition layer.

5.3.2. $P_{2}$ analysis. The same analysis with a standard $P_{2}$ method instead of a $P_{1}$ method shows that again half an order of rate of convergence is lost in the transition layer. For an enriched $P_{2}$ method, the shape functions on the transition layer $[0, h]$ are

$$
\frac{(x-h)(2 x-h)}{h^{2}}, \quad \frac{x(2 x-h)}{h^{2}}, \quad \frac{4 x(h-x)}{h^{2}} \text { and } \quad\left(1-\frac{x}{h}\right) f(x)
$$

The interpolation function is defined on $[0, h]$ by

$$
f^{h}(x)=\left(1-\frac{x}{h}\right) f(x)+\frac{x}{h^{2}}(2 x-h) f(h)+\frac{4 x(h-x)}{h^{2}} f\left(\frac{h}{2}\right)
$$

and the interpolation error on the transition layer may be estimated as follows:

$$
\int_{0}^{h}\left|f^{h}(x)-f(x)\right|^{2} \mathrm{~d} x \leqslant C h^{5}
$$


One can conclude that the $L^{2}$-norm on the global domain including the transition layer will be bounded by $C h^{5 / 2}$ instead of $C h^{3}$ for a standard $P_{2}$ method on a smooth function.

Even though the latter analysis is not a rigorous proof of the lost of half an order because of the transition layer, it seems to corroborate the experimental numerical results. This onedimensional analysis generalizes the one in Reference [22] for non-polynomial functions.

\subsection{Back to PUFEM}

The previous analysis and the numerical results show a lack of accuracy due to the transition layer with XFEM. This method is originally based on a partition of unity principle. However, the original work by Melenk and Babuška [6] shows that PUFEM (partition of unity finite element method) does not exhibit such a lack of accuracy. Thus, an idea is to be closer to PUFEM original principle.

The domain is divided into two overlapping sub-domains $\Omega_{1}$ and $\Omega_{2}$ (see Figure 17), one of them $\left(\Omega_{2}\right)$ containing the crack tip. Let $\psi_{1}$ and $\psi_{2}$ be a corresponding Lipschitz-continuous partition of unity on $\Omega$, i.e.

$$
\psi_{1}(x)+\psi_{2}(x)=1 \quad \text { on } \Omega, \quad \operatorname{Supp}\left(\psi_{i}\right) \subset \Omega_{i} \quad \psi_{i}(x) \geqslant 0 \quad \text { on } \Omega
$$

Let us consider an approximation space $V_{1}^{h}$ on $\Omega_{1}$ and an approximation space $V_{2}^{h}$ on $\Omega_{2}$ having an optimal convergence rate (which means that $V_{2}^{h}$ is enriched with singular functions). Then it is possible to define the following PUFEM on $\Omega$ :

$$
V_{h}=\left\{v^{h}=\psi_{1} v_{1}^{h}+\psi_{2} v_{2}^{h}: v_{1}^{h} \in V_{1}^{h}, v_{2}^{h} \in V_{2}^{h}\right\}
$$

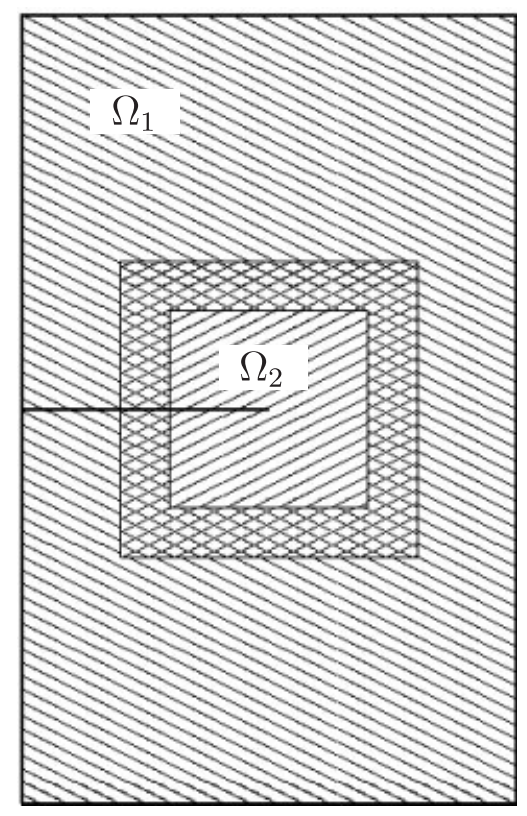

Figure 17. Decomposition in two overlapping sub-domains $\Omega_{1}$ and $\Omega_{2}$. 
Let us assume that $u \in H^{1+\alpha}\left(\Omega ; \mathbb{R}^{2}\right)$, there exist $u_{1}^{h} \in V_{1}^{h}$ and $u_{2}^{h} \in V_{2}^{h}$ with

$$
\left\|u_{1}^{h}-u\right\|_{1, \Omega_{1}} \leqslant C_{1} h^{\alpha}\|u\|_{1+\alpha, \Omega_{1}}, \quad\left\|u_{2}^{h}-u\right\|_{1, \Omega_{2}} \leqslant C_{2} h^{\alpha}\|u\|_{1+\alpha, \Omega_{2}}
$$

Then, considering $u^{h}=\psi_{1} u_{1}^{h}+\psi_{2} u_{2}^{h}$, one has

$$
\begin{aligned}
\left\|u^{h}-u\right\|_{1, \Omega}= & \left\{\int_{\Omega}\left\|\psi_{1}\left(u_{1}^{h}-u\right)+\psi_{2}\left(u_{2}^{h}-u\right)\right\|^{2} \mathrm{~d} x+\int_{\Omega} \| \nabla \psi_{1}\left(u_{1}^{h}-u\right)^{\mathrm{T}}\right. \\
& \left.+\nabla \psi_{2}\left(u_{2}^{h}-u\right)^{\mathrm{T}}+\psi_{1}\left(\nabla u_{1}^{h}-\nabla u\right)+\psi_{2}\left(\nabla u_{2}^{h}-\nabla u\right) \|^{2} \mathrm{~d} x\right\}^{1 / 2}
\end{aligned}
$$

Hence, the following estimate holds

$$
\left\|u^{h}-u\right\|_{1, \Omega} \leqslant \sum_{i}\left\|u_{1}^{h}-u\right\|_{1, \Omega_{i}}+\max _{i}\left(\left\|\nabla \psi_{i}\right\|_{\infty, \Omega}\right) \sum_{i}\left\|u_{i}^{h}-u\right\|_{0, \Omega_{1} \cap \Omega_{2}}
$$

Finally we obtain

$$
\left\|u^{h}-u\right\|_{1, \Omega} \leqslant C h^{\alpha}\|u\|_{1+\alpha, \Omega}
$$

This shows that the optimal convergence rate is obtained.

Let us remark that this method is different from XFEM-d.g. only on the transition layer: in XFEM-d.g., the function $\psi_{2}$ is missing. This problem is also present with the classical XFEM. Note also that the size of the transition layer does not influence the estimate (if $\delta$ is the width of the transition layer, the term $\max _{i}\left(\left\|\nabla \psi_{i}\right\|_{\infty, \Omega}\right)$ in (10) can be chosen in $1 / \delta$, but the term $\sum_{i}\left\|u_{i}^{h}-u\right\|_{0, \Omega_{1} \cap \Omega_{2}}$ is of order $\left.\delta h^{\alpha}\right)$.

\subsection{Pointwise matching (XFEM-p.m.)}

Since, in the latter section, it has been observed that the width of the transition layer has no influence on the convergence rate, a transition layer with a vanishing width should be convenient (i.e. with $\Omega_{1}$ and $\Omega_{2}$ a partition of $\Omega$ ). Of course, doing so, the finite element method will be no more conformal and one has to choose the kind of matching to prescribe (integral matching, pointwise matching, mortar, etc.).

We test XFEM-d.g. with a pointwise matching condition. Assuming that $\Omega_{2}$ is a union of the triangles in the enriched area, the approximate displacement $u_{h}$ is such that $u_{h}=u_{h}^{1}$ on $\Omega_{1}$ and $u_{h}=u_{h}^{2}$ on $\Omega_{2}$, where

$u_{h}^{1}=\sum_{i \in I\left(\Omega_{1}\right)} a_{i} \varphi_{i}+\sum_{i \in I_{H}\left(\Omega_{1}\right)} b_{i} H \varphi_{i}, \quad u_{h}^{2}=\sum_{i \in I\left(\Omega_{2}\right)} a_{i} \varphi_{i}+\sum_{i \in I_{H}\left(\Omega_{2}\right)} b_{i} H \varphi_{i}+\sum_{j=1}^{4} c_{j}^{1} F_{j} e_{1}+\sum_{j=1}^{4} c_{j}^{2} F_{j} e_{2}$

and $u_{h}^{1}=u_{h}^{2}$ on each finite element node lying on the interface $\Gamma_{12}=\bar{\Omega}_{1} \cap \bar{\Omega}_{2}$. This is in fact equivalent to extend the singular functions on the layer of elements outside the enrichment area with polynomials (see Figure 18, compared to Figure 13). 


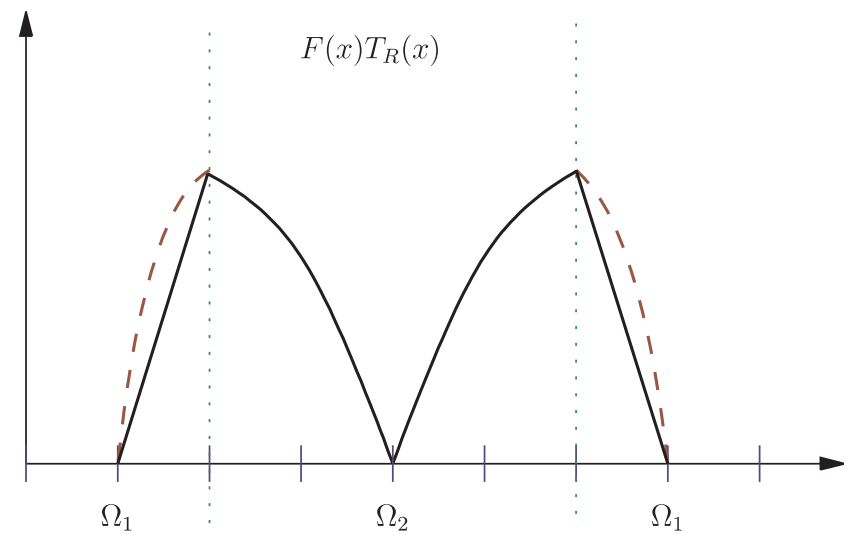

Figure 18. Truncation of the singular enrichment function $F$ for a $P_{1}$ XFEM with a pointwise matching condition (XFEM-p.m.).

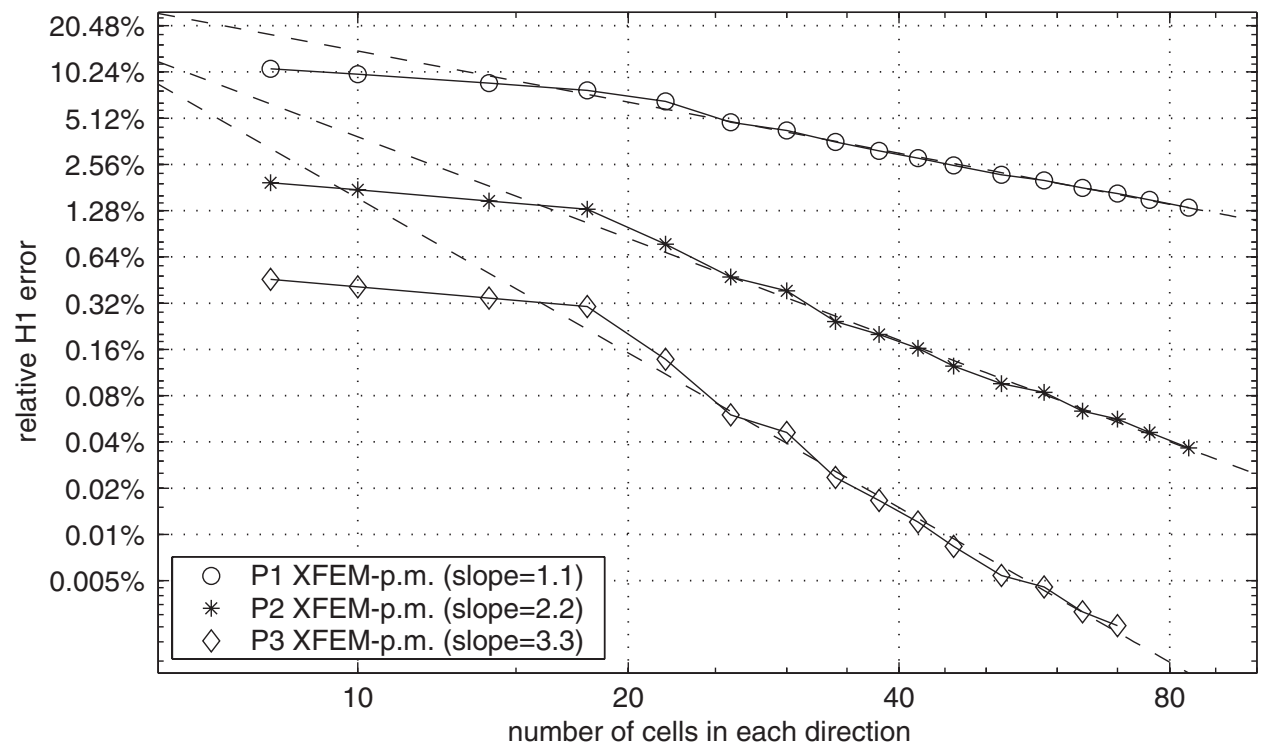

Figure 19. Convergence of XFEM with pointwise matching condition for mode I problem.

The new convergence curves (Figure 19) are now optimal (actually, with a slight superconvergence). The body deformation is shown in Figure 20 for degree three polynomials. One observes very smooth Von Mises stress iso-values, despite the non-conformity, compared to Figure 8 for the classical XFEM. 

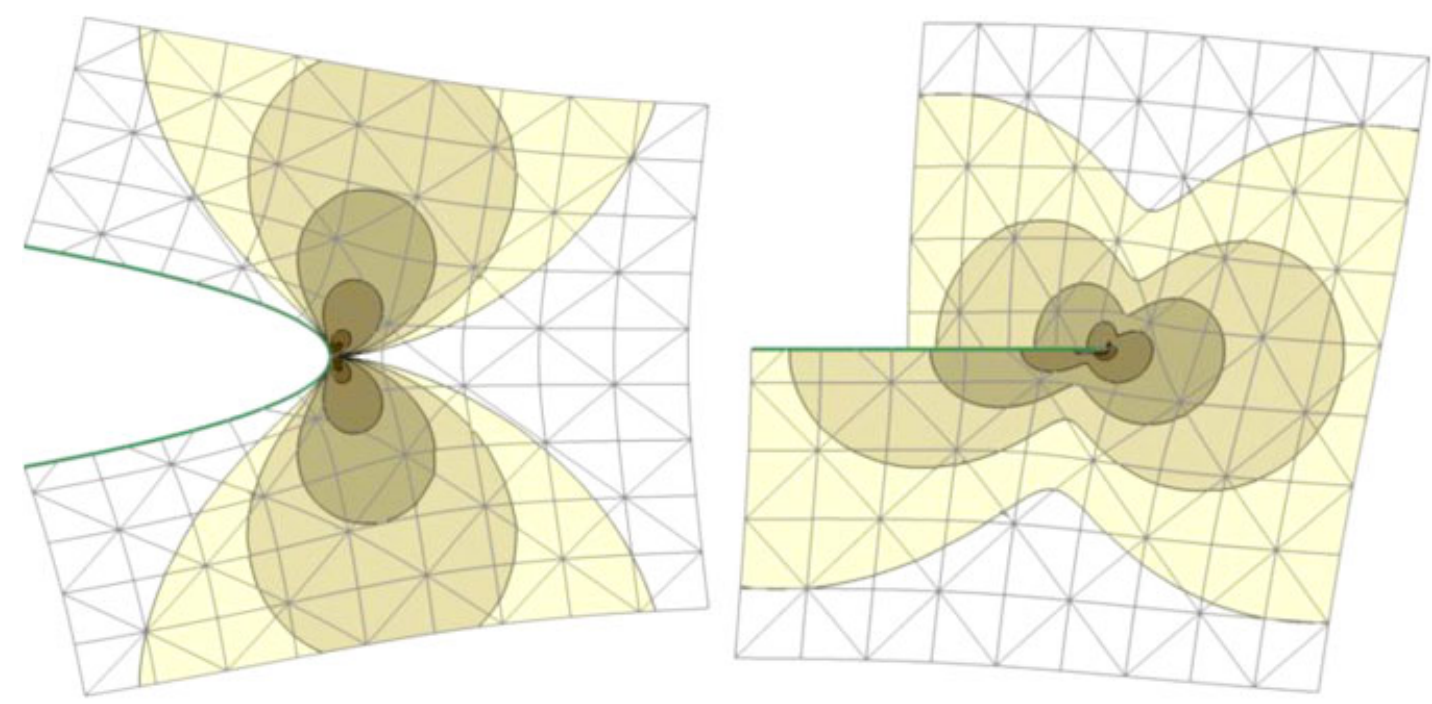

Figure 20. $P_{3}$ XFEM solutions for mode I and mode II problems with a pointwise matching condition (contour levels of Von Mises stress). To be compared with Figure 8.

\section{WEAKER SINGULARITIES}

Finally, we build a new test problem combining a regular solution, mode I and mode II displacements, $u_{\mathrm{I}, 3 / 2}$ or $u_{\mathrm{II}, 3 / 2}$ which are the next opening modes in the crack tip displacement in expansion (1):

$$
\begin{aligned}
& u_{\mathrm{I}, 3 / 2}=\frac{4}{3 \lambda+3 \mu} r^{3 / 2}\left(\begin{array}{c}
\cos \frac{\theta}{2}\left[(3 \lambda+7 \mu) \cos ^{2} \frac{\theta}{2}-3 \lambda-6 \mu\right] \\
\sin \frac{\theta}{2}\left[(\lambda+5 \mu) \cos ^{2} \frac{\theta}{2}-\lambda-2 \mu\right]
\end{array}\right) \\
& u_{\mathrm{II}, 3 / 2}=\frac{-4}{3 \lambda-\mu} r^{3 / 2}\left(\begin{array}{c}
\sin \frac{\theta}{2}\left[(7 \lambda+11 \mu) \cos ^{2} \frac{\theta}{2}-\lambda-2 \mu\right] \\
\cos \frac{\theta}{2}\left[(3 \lambda-\mu) \cos ^{2} \frac{\theta}{2}-3 \lambda-2 \mu\right]
\end{array}\right)
\end{aligned}
$$

A representation of these two opening modes can be found in Figure 21. Let us just remark that this additional singularity belongs to $H^{5 / 2-\varepsilon}\left(\Omega ; \mathbb{R}^{2}\right)$ for any $\varepsilon>0$.

The numerical results we obtain with different versions of XFEM are given on Figure 22. First, we observe that, without any enrichment, the convergence rate is $O(\sqrt{h})$ whatever the degree of the polynomials is. This result was expected as the main singularity belongs to $H^{3 / 2-\varepsilon}\left(\Omega ; \mathbb{R}^{2}\right)$. Then, for the 'pointwise matching' XFEM version, the convergence rate is equal to 1 when we use $P_{1}$ finite element functions, but it remains limited to $O\left(h^{3 / 2}\right)$ with higher order polynomials. This is the expected result since mode I and mode II singularities are taken into account by our approach. It is not the case for the additional singularity. The latter 



Figure 21. Contour levels of the Von Mises stress for the two $r^{3 / 2}$ opening modes $u_{\mathrm{I}, 3 / 2}$ and $u_{\mathrm{II}, 3 / 2}$.



Figure 22. Convergence for an analytical solution $u_{\mathrm{I}}-3 u_{\mathrm{II}}-5 u_{\mathrm{I}, 3 / 2}+P(x)$ where $P(x)$ is a regular solution on the non-cracked domain.

belongs to $H^{5 / 2-\varepsilon}\left(\Omega ; \mathbb{R}^{2}\right)$, so one can expect the theoretical convergence order to be limited to $h^{3 / 2}$, for a sufficient degree of the polynomials. Finally, the only surprising result concerns the use of XFEM with a fixed enrichment area (see Section 4). In this case, we obtain really better results than with the previous one. 
To conclude this section, let us make two remarks. First of all, when high-order polynomials are used, the suitable enrichment functions must be added if we want to reach an optimal convergence order. In the previous test, we should also add the following functions for each direction (coming from (11)):

$$
r^{3 / 2} \cos \frac{\theta}{2}, \quad r^{3 / 2} \sin \frac{\theta}{2}, \quad r^{3 / 2} \cos \frac{\theta}{2} \cos ^{2} \frac{\theta}{2}, \quad r^{3 / 2} \sin \frac{\theta}{2} \cos ^{2} \frac{\theta}{2}
$$

Let us remark that it means we should add only eight degrees of freedom, which is not expensive.

The use of XFEM with a fixed enrichment area seems to be more flexible when the exact singularity is not added. This opens an interesting field of investigation in the use of XFEM in the case where the singularities are not analytically known or if they are too complicated to be used. The challenge is to define a set of functions which reasonably represent frequently encountered singularities.

\section{STRESS INTENSITY FACTORS}

The crack growth criteria requires the computation of the stress intensity factors (SIF) $K_{\mathrm{I}}$ and $K_{\mathrm{II}}$. Those two numbers correspond to the amplitude of the two opening modes (2) and (3). A standard way to compute these stress intensity factors is to use the well-known J-integral and the interaction integral. It leads to good estimations of the SIF since it involves evaluations of integrals on a ring of elements centred on the crack tip, thus avoiding numerical difficulties related to the singular nature of the near crack tip displacement. More details can be found in References [12,27, 28].

The experimental convergence results are presented in Figure 23, the exact solution is $4 u_{\mathrm{I}}-3 u_{\mathrm{II}}+P(x)$, where $P(x)$ is a regular solution on the non-cracked domain. The curves for $K_{\mathrm{I}}$ and $K_{\mathrm{II}}$ are very similar. The level of error for the two intensity factors is quite small (the error for the coarsest mesh is less than 7\%).

While the curves of the classical $P_{k}$ finite element method are very smooth, and show a constant convergence rate equal to 1 , the curves of XFEM-p.m. are much more noisy. This difference may be explained by the fact that classical $P_{k}$ finite element method did always overestimate the magnitude of the SIF in our tests, while XFEM-p.m. was oscillating around the exact values.

However, the convergence rates for the $P_{k}$ XFEM-p.m. appear to be much better, they are approximately equal to $k+1$. Numerical experiments show that the presence of the $r^{3 / 2}$ singularities does not seem to affect the convergence rate of the SIF.

\section{CONCLUDING REMARKS AND PERSPECTIVES}

Special attention was devoted to the rate of convergence of the XFEM method. Different factors of deterioration were pointed out in the classical XFEM method and some improvements were suggested. A first modification is relative to the step function of enrichment used to represent the jump in the displacement field across the crack. The corresponding partition of unity must be of the same degree than the finite element polynomials, so that the displacement may be 

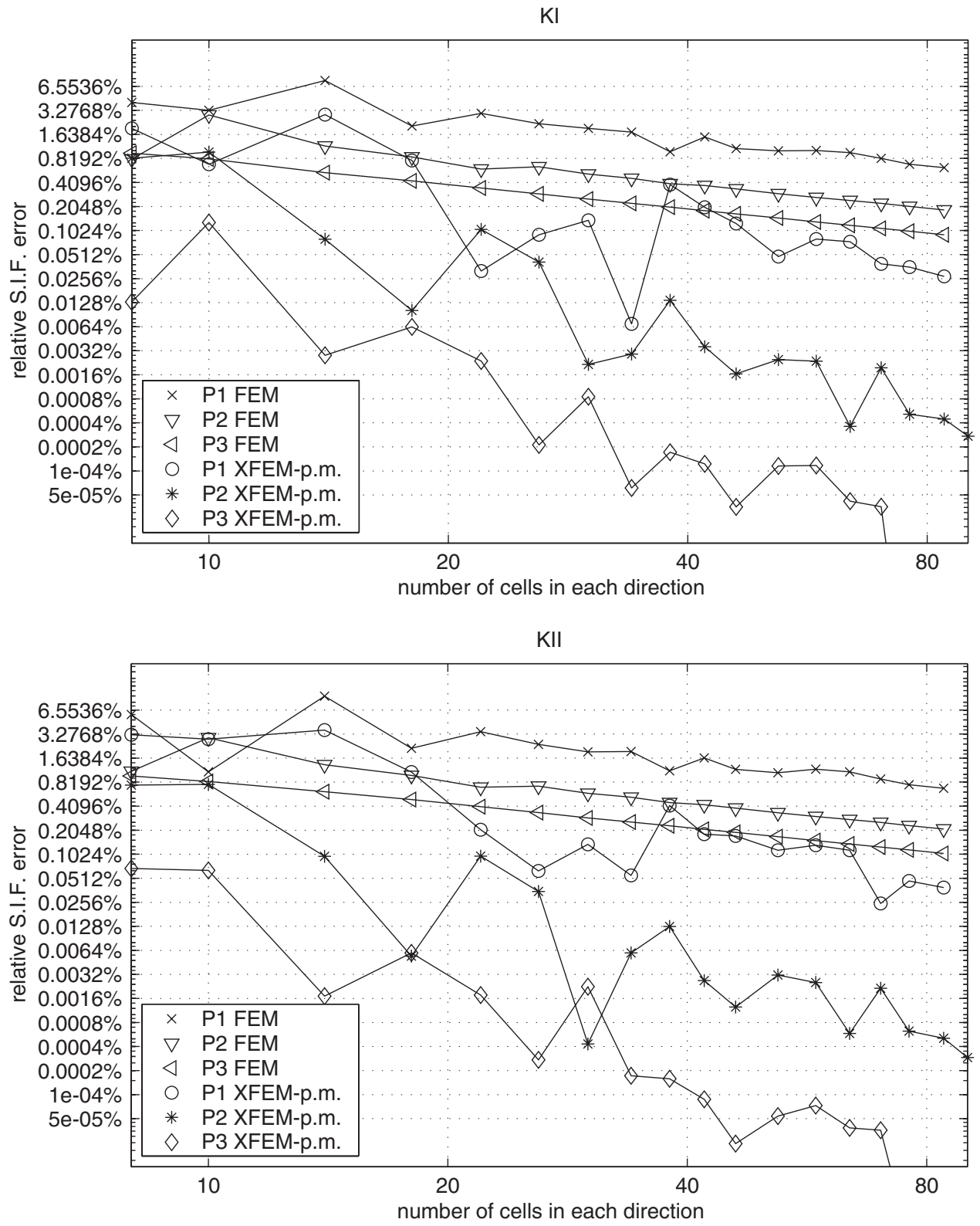

Figure 23. Convergence of $K_{\mathrm{I}}$ and $K_{\mathrm{II}}$ for a mode I + mode II + regular problem.

approximated in a satisfactory way along the crack. The second improvement is related to the asymptotic near-tip displacement solutions in the XFEM basis. The enrichment must concern a whole zone around the crack tip, independent of the mesh parameter $h$. Moreover, it is necessary to correctly take into account the transition between the enriched zone and the other 
one, either using a partition of unity method, or using a non-conformal method. Concerning the integration of the singularities, a simple and efficient quadrature rule has been proposed and evaluated. These improvements were applied to solve plane elasticity crack problems and the optimal rate of convergence was numerically verified.

The extension of this work to tridimensional problems is an open question, since the singularities are difficult to express. Nevertheless, the extension to plates and shells should be more straightforward and is a work in progress.

The numerical experiments were done with the finite element library Getfem ++ [29]. Test programs are available in the distribution.

\section{ACKNOWLEDGEMENTS}

The authors would like to thank J. P. Lambelin, J. J. Pesqué and B. Toson from CEA/CESTA for several motivating and helpful discussions.

\section{REFERENCES}

1. Anderson TL. Fracture Mechanics: Fundamentals and Applications (2nd edn). CRC Press: Boca Raton, FL, 1994.

2. Shah SP. Fracture Mechanics of Concrete: Applications of Fracture Mechanics to Concrete, Rock and Other Quasi-Brittle Materials. Wiley: New York, 1995.

3. Sun TC. Mechanics of Aircraft Structures. Wiley: New York, 1998.

4. Strang G, Fix GJ. An Analysis of the Finite Element Method. Prentice-Hall: Englewood Cliffs, NJ, 1973.

5. Babuška I, Caloz G, Osborn J. Special finite element methods for a class of second order elliptic problems with rough coefficients. SIAM Journal on Numerical Analysis 1994; 31:945-981.

6. Melenk JM, Babuška I. The partition of unity finite element method: basic theory and applications. Computer Methods in Applied Mechanics and Engineering 1996; 139:289-314.

7. Babuška I, Melenk JM. The partition of unity method. International Journal for Numerical Methods in Engineering 1997; 40:27-758.

8. Duarte CAM, Babuška I, Oden JT. Generalized finite element method for three-dimensional structural mechanics problems. Computers and Structures 2000; 77:219-232.

9. Strouboulis T, Babuška I, Copps T. The design and analysis of the generalized finite element method. Computer Methods in Applied Mechanics and Engineering 2000; 181:43-69.

10. Strouboulis T, Copps K, Babuška I. The generalized finite element method. Computer Methods in Applied Mechanics and Engineering 2001; 190:4081-4193.

11. Babuška I, Banerjee U, Osborn JE. Survey of meshless and generalized finite element methods: a unified approach. Acta Numerica 2003; 1-125.

12. Moës N, Dolbow J, Belytschko T. A finite element method for crack growth without remeshing. International Journal for Numerical Methods in Engineering 1999; 46:131-150.

13. Belytschko T, Black T. Elastic crack growth in finite elements with minimal remeshing. International Journal for Numerical Methods in Engineering 1999; 45(5):601-620.

14. Dolbow J, Moës N, Belytschko T. An extended finite element method for modeling crack growth with frictional contact. Computer Methods in Applied Mechanics and Engineering 2001; 190:6825-6846.

15. Sukumar N, Moës N, Moran B, Belytschko T. Extended finite element method for three dimensional crack modelling. International Journal for Numerical Methods in Engineering 2000; 48:1549-1570.

16. Belytschko T, Moës N, Usui S, Parimi C. Arbitrary discontinuities in finite elements. International Journal for Numerical Methods in Engineering 2000; 50(4):993-1013.

17. Gravouil A, Moës N, Belytschko T. Non-planar 3D crack growth by the extended finite element and level sets. Part II: level set update. International Journal for Numerical Methods in Engineering 2002; 53(11): 2569-2586.

18. Sukumar N, Chopp DL, Moës N, Belytschko T. Modeling holes and inclusions by level sets in the extended finite element method. Computer Methods in Applied Mechanics and Engineering 2001; 90(46,47):6183-6200. 
19. Moës N, Belytschko T. Extended finite element method for cohesive crack growth. Engineering Fracture Mechanics 2002; 69(7):813-833.

20. Moës N, Gravouil A, Belytschko T. Non-planar 3D crack growth by the extended finite element and level sets. Part I: mechanical model. International Journal for Numerical Methods in Engineering 2002; 53(11):2549-2568.

21. Stazi FL, Budyn E, Chessa J, Belytschko T. An extended finite element method with higher-order elements for curved cracks. Computational Mechanics 2003; 31:38-48.

22. Chessa J, Wang HW, Belytschko T. On construction of blending elements for locally partition of unity enriched finite element methods. International Journal for Numerical Methods in Engineering 2003; 57(7): 1015-1038.

23. Grisvard P. Singularities in Boundary Value Problems. Masson: Paris, 1992.

24. Lemaitre J, Chaboche J-L. Mechanics of Solid Materials. Cambridge University Press: Cambridge, 1994.

25. Sukumar N, Préévost J-H. Modeling quasi-static crack growth with the extended finite element method, part I: computer implementation. International Journal of Solids and Structures 2003; 40:7513-7537.

26. Béchet E, Minnebo H, Moës N, Burgardt B. Improved implementation and robustness study of the X-FEM method for stress analysis around cracks. International Journal for Numerical Methods in Engineering, 2005, in press.

27. Yau J, Wang S, Corten H. A mixed-mode crack analysis of isotropic solids using conservation laws of elasticity. Journal of Applied Mechanics 1980; 47:335-341.

28. Destuynder Ph, Djaoua M. Mathematical Methods in the Applied Sciences 1981; 3:70-87.

29. Pommier J, Renard Y. Getfem ++. An open source generic C++ it library for finite element methods, http://www-gmm.insa-toulouse.fr/getfem. 